see Miss B., age 26 years. She had been treated by tonics, creosote and the same old treatment with which we are all familiar. She had been ill for six months. This patient was almost dead. The microscope showed tubercular bacilli in abundance. Her pulse 150, temperature $104 \frac{1}{2}$, examination of lungs showed upper lobes of both badly affected from apex down. Severe night sweats, and almost an absence of digestion with such ferment in her bowels that she suffered severely with them. Her whole system was saturated with tuberculosis. So bad was this case that I thought she would die before two weeks, and considered it useless to treat the case as others had given her up. On the earnest solicitations of her relatives I began treating her; using anti-tubercular eerum, and at the end of four days had the amount of serum per dose up to twenty drops, which is double the amount for that time in ordinary cases. She then began to feel better. I placed her on intestinal antiseptics. Her digestion improved and the night sweats ceased entirely. At the end of the firet month she was able to take a ride every day, if the weather would permit. During the second month she had a relapse on account of a small abscess forming and breaking in the right lung. She again improved. At the end of three months she took the measles; this was serious for her, but she came through all right. At the end of four months she went to the country, and is using the antitubercular serum per rectum. She is still living and feeling well, but not improving. Her pulse came down to 80 and 90 from 130 to 150 , temperature normal of mornings, and 100 to $100^{\frac{1}{2}} / 2$ evenings, and at times normal of evenings. Digestion good, appetite good. I had a decided effect in this case in assisting the serum treatment by the use of sat. sol. of borat. sod. one ounce each day per rectum, a tablespoonful three times a day internally. I think this a flne addition in the treatment of tubercular diseases.

I have selected these twelve cases for report, as they are about average cases with me. To recapitulate:

Five cases - third stage.-One dead; one benefited some; one greatly benefited; one well: one living six months after being given up to die at any time.

Three cases-second stage.--One greatly improved; one well over one year; one improving fast.

Four cases-first stage.--Four well.

I advise tonics, cod-liver oil, antiseptics sprayed in the throat and lungs of boro-lyptol, listerin, etc. I advise two or three times a week an enema or normal solution of sod. chlorid per rectum and flush the colon. This acts as a disinfectant, and the absorption of a portion of this solution in the circulation is very beneficial to the patient. Then I wish to impress the good of using sat. solut. of borat. sod., tablespoonful internally three times a day, and about an ounce injected each day in the rectum. When pus exists in the sputum, I advise the use of anti-streptococcus, alternating with the anti-iubercular serum, and the effect is at once marked.

My success has been so great in many other cases and I feel that I can almost insure a case in the first, or incipient stage, where the cavities are small, that many will improve, and at times recover even in the second stage, and occasionally in the third stage.

\section{MODERN METHODS IN THE TREATMENT OF TUBERCULOSIS.}

Presented to the Section on Practice of Medicine at the Forty-ninth Annual Meting of the American Medical Association, held at Denver, Colo., June 7-10, 1898.

BY WILLIAM F. WAUGH, A.M., M.D.

PROFESSOR OF PRACTICE OF MEDTCINE, ETC., ILIINOIS MEDICAL COLERGE.

If we believe that for every ill that affects humanity there is somewhere a remedy, it would seem that even through the operation of blind chance there was a fair opportunity for lighting upon the means of curing tuberculosis. For in all parts of the medical to world there is put into practice a most miscellaneous assortment of experiments. The power of suggestion is being demonstrated by the claims made in behalf of nearly all these essays. The need is so terrible, the boon to humanity would be so enormous, that one must look with kindly sympathy upon the man who desires to succeed.

The fact that success in this matter means so much to humanity demands from us most careful scrutiny that no really valuable idea shall be lost on the most critical examination, that no delusion shall be permit ted to prevail. Should my remarks, therefore assume somewhat the shape of criticism, it is with no unsympathetic or pessimistic spirit, but with the earnest desire to favor the truth.

First, we will consider the climatological aspect of the question. When we inhale the pure breezes of Colorado we do not wonder at the enthusiasm that prevails among the residents as to its salubrity. We recognize at once that this is founded upon reason, not upon commercial scheming. We have sent our patients to the Denver region for years, and can readily recall notable instances of its restoration or even curative effects. But that all cases are not thus benefited is sadly attested by the monuments in yonder populous city of the dead. We trust that the physicians of Denver, who have such exceptional opportunities for the study of the question, will give us clean and definite instructions as to what cases should be sent here, and where to send the others.

At present we have some valuable but scanty data upon which to base our advice. Incipient cases do well here, as they do in most places; the best results are obtained in these instances in which the diagnosis is inferential rather than positive. But hemorrhagic cases ascend to this altitude with peril. They fare badly here as well as at the seashore; so that their destination is problematic. Beside the conditions of the case, there lies behind this a consideration as to the personality of each patient. The effects of climate are not now believed to reside in the power of any atmosphere to starve or to poison the microorganisms. That climate is best for each person, that most conduces to his physical health, that allows him to live the most wholesome life as an animal, usually, in the open air, with sunshine, and free ventilation.

Broadly speaking, mankind is divisible into two classes. Types of both may be found in the same family. If one feels strong and vigorous in the pure, cool thin air of the hills, to the hills he should go; but if he feels bad here, and experiences a sense of comfort, of exhilaration, of renewed vitality when he smells the damp air of the salt meadows, he will there have the best chance for cure. Beyond this, and the injunction to keep as far as possible from other cases of tuberculosis, there is little of certainty in the choice of a climate. As to the effects of any atmos. phere upon cases of tubercular, streptococous, staphylococcus and mixed infection, upon primary microbic affections and these engrafted upon previous inflam. matory processes, we have as yet but a feeble light. The hope of securing more has brought many of us to Denver.

Of the various forms of serum therapy, I shall say but little, as the program is filled with numerous papers by those who are better qualified to speak. Be they successes or failures each marks an approximation to the truth, if it be but by clearing the way for further experiment. They are commendable in that they are based upon a study of the phenomena of the disease and of the conditions noted in the biology of the 
micro-organisms present. Surely if success be obtainable, it is in this direction.

In this connection I will present some facts worth considering. Tuberculosis is separated from the large majority of microbic affections in that it is not self. limited or self-protective. Tuberculosis is also distinguished from the majority of such affections in that we do not find leucocytosis attending it. Can we correlate these two facts, and infer that the failure of self-immunization is due to the fact that nature has here failed to combat this disease by a multiplication of her defenders, the leucocytes? If, so, we may find value in that singular discovery of Vaughan, that nuoleinic acid increases the number and activity of the white blood corpuseles. This hypothesis, pregnant with possibilities that may change the science of therapeutics and vitally modify the history of the human race, is all the more worthy of commendation in that it brings us back to the truest basis of therapy, the study of the physiologic conditions aberrant in disease and utilizable in its treatment. The finality of therapeutics must be the comprehension, restoratiou and maintenance of a normal physiologic state.

But vastly important as are the germicidal elements, and the value of climate as a means of reinforcing the vitality to the point of rendering it victorious, they are by no means our only weapons. The armamen. taria with which we relieve the various symptoms of tuberculosis require no mention on my part; but I wish to speak of two agents that have served me well. Many years ago I heard Samuel H. Dickson say that when we learn to control the digestive disorders of consumption we would cure that disease. I need not more than refer to the difficulty of feeding these patients, the anorexia, dyspepsia, constipation and diarrhea, malassimilation, etc, marking their history. But all these disappear under the use of calcium sulfocarbolate, and with them from one to three degrees of the fever, and in all, from 30 to 60 per cent. of the sum total of the symptoms and phenomena is removed by this single expedient. These results have followed so uniformly that I feel warranted in suggesting a trial of this salt. I have given it daily in doses of 2 and 6 grains for nearly two years without any discernible objectionable effects.

The other agent is iodoform. In some consumptives there appears to be a tolerance of this drug, as they have taken from 10 to 20 grains daily for months without any symptom of iodism, while this unpleas ant condition has followed the use of four grains daily in non-tuberculous individuals. Whether its effects be germicidal, absorbent or simply those of a pulmonary and analgesic, or of all three combined, the use of this remedy has given me better results than any other pulmonary germicide.

\section{THE PSYCHOLOGY OF HABITUAL CONSTIPATION.}

Presented to the Section on the Practice of Medicine at the Fortr-ninth Annual Meeting of the American Medical Association, held at Denver, Colo., June $7-10,1898$.

BY ALBERT H. BURR, Pн.B., M.D.

ADJUNCT PROFESSOR OF PRACTICE OF MEDICINE COLLEGE OF PHYSICIANS AND SURGEONS, ATTENDING PHYSICIAN PROVIDENT HOSPITAL. CHICAGO, ILL.

Among the great phenomena of nature one of the most interesting is that of the rhythmic action of her forces in obedience to ever constant laws. The cycle of darkness and light that measures a day; of recurring seasons that calendar a year; of ocean's tidal ebb and flow that mark her throbbing pulse: all these are but so many demonstrations of a universal law of rhythm. In our bodies similar laws of periodicity are essential to organic health or even to life itself. Restful sleep must follow wakeful toil in just proportion and at proper intervals. Respiration and circulation are dependent upon ever constant, regulated muscular impulses. The processes of digestion, assimulation and excretion obey this same law of rhythm. So long as these periodic forces are undisturbed in their rhythm by careless or vicious habits of the individual, or by accidental and pathologic changes beyond his control, healthy, organic functions are assured.

Mental control of rhythmic functions.-The control of these rhythmic forces is for the most part, outside of and beyond our conscious mental appreciation. They act with automatic precision whether we wake or sleep, little influenced by our wills, our desires or our reasoning faculties. They are said to be controlled by the great sympathetic nervous system which connects the various organs of the body in their functions, with ganglionic and cerebro-spinal centers. This is not true in a literal sense, for this wonderful system is itself only a mechanism; an intricate co-ordination of communicating lines, sensitized for the transmission of impressions to and orders from a central governing intelligence. Whether we accept the theory of a dual mind or not, we are forced to the conclusion that this marvelous labyrinth of communicating sympathetic nerves and the organs to which they are distributed, and hence the functions of these organs themselves are dominated by a never sleeping, ever acting intelligent entity.

Since we know these functionating organs and their special nervous systems act for the most part independently of our objection primary consciousness and volition, we ara impelled to the belief that they are controlled by a sub-conscious, instinctive faculty of the mind which regulates all the automatic move. ments of the nun.striated muscles; that it presides over the functions of every organ of our bodies, and that it is capable of being reached and directed itself by psychic influences from within and without. To show this psychic relation and control over the peristaltic action of the bowels and the function of defeca. tion, as well also the practicability of relieving habitual constipation, of non-pathologic origin, by psychic methods are the problems set before us in this paper. We are confident, if their solutions are made evident, a flood of light will be thrown upon many other functional derangements and their rational treatment made plain; a treatment too little appreciated and too often flippantly ridiculed and dismissed by thousands of good physicians who have not investigated the worth of suggestive therapeutics.

Peristalsis and dof'cation.-The essential physical element in the act of defecution is peristalsis. A clear understanding of this function is of vital importance in this discussion. Peristalsis may be defined as a peculiar rhythmic contraction of successive muscular fibers of the intestine. This undulating movement extends through the length of the canal and is called the peristaltic wave. It is less active in the large than in the small intestine. Its function in the upper bowel is, 1, to assist in mixing the food from the stomach with bile and the digestive ferments of the pancreas and intestinal glands; 2, to bring nutrient matter in contact with large absorbing surfaces, and 